\title{
Improving Bluetooth Mesh Energy Efficiency Using Clustering
}

\author{
Joelton Deonei Gotz, Ohara Kerusauskas Rayel and Guilherme Luiz Moritz
}

\begin{abstract}
This work proposes a Clustering Algorithm to improve the energy efficiency of Bluetooth Mesh networks. To further reduce the burden over the Cluster Heads, a Radio Duty Cycling algorithm that requires only a simple modification on the Bluetooth packet transmission logic is proposed. Computer simulations show that the radio duty cycling and clustering methods are effective in improving energy efficiency. It is observed that duty cycling provides a $78 \%$ improvement on the energy efficiency. In addition, simulations show that the proposed clustering technique is effective in controlling the excessive message replication that is inherent in flooding operation, which in turn have a positive impact on packet delivery ratio (PDR) and network scalability. Finally, it can be observed that the proposed clustering algorithm together with the proposed radio duty cycling algorithm can provide an improvement on the energy efficiency when compared to the baseline Bluetooth Mesh profile.
\end{abstract}

Index Terms-Bluetooth Mesh, LEACH, Energy Efficiency, Flooding Routing

\section{INTRODUCTION}

$\mathbf{T}$ here is a consensus either on industry or academia that the future of Internet is the ubiquitous connectivity between everything that can benefit of such feature. For this reason, we have been experiencing an explosion of papers and applications about the so-called Internet of Things. Every year, forecasts of several institutes like Forbes [1], IDC [2] and Ericsson [3] predict a growing market trend and an explosion in the number of connected devices. It is expected that IoT numbers surpass the trillion dollar barrier on Worldwide technology spending and the 28 billion barrier in the number of connected devices by 2022. The same cited reports show that, in 2018 there were already 20 billion connected objects on the Internet, which represents a worldwide market of more than U\$700 billion.

On the other hand, we still do not experience a fully connected world. There are a lot IoT use cases, like building automation systems, Smart Grid initiatives, users with Smartphones and Smartwatches and industrial plants connected to the Internet, but a qualitative difference in our lifestyle will be only noticed when the density of the deployed sensors reaches levels that are the double or even triple of what is experienced today [4]. To reach this level of deployment, some issues must still be addressed. Internet of Things devices are heterogeneous, ranging from very small body implantable devices to big devices like cars or white goods. Each of these

Joelton Deonei Gotz, Ohara Kerusauskas Rayel and Guilherme Luiz Moritz are with PPGSE/UTFPR, Curitiba/PR, 80230-901, Brazil (e-mail: joelton_jdg@hotmail.com, \{oharakr,moritz\}@utfpr.edu.br).

Digital Object Identifier: 10.14209/jcis.2021.17 things has a different communication requirement, like range, power consumption, number of connected nodes, maintenance costs and reliability levels.

For this reason, several protocols were proposed to fulfill different use cases. For example, a widely accepted medium access control protocol for industrial applications is the IEEE Time Switching Channel Hopping (TSCH) [5], [6]. In TSCH network nodes are synchronized and time is split into time slots. Transmission and reception are coordinated by a schedule that repeats over time. For Smart Cities and Smart Metering applications, which are tolerant to small data rates and high latency, low power wide area (LPWAN) technologies were developed like LoRaWAN [7] and SigFox [8]. These applications offer different tradeoffs than TSCH that are not ideal to connect low power devices distributed over large geographical areas. LPWAN networks are intended to transmit at very low data rate (hundreds of bytes per second) throughout a range of several kilometers. For office or home automation applications, several technologies were proposed where the most prominent are Thread [9], Zigbee [10], Wi-Fi [11] and Bluetooth [12]. Thread and Zigbee are based on IEEE 802.15.4 [13] standard which specifies a low power transmission scheme that can have its range virtually extended through mesh routing. Wi-Fi and Bluetooth, on the other hand, have some disadvantages over 802.15.4 based protocols, for example, Wi-Fi was originally released with focus in high data rate, and not for low power consumption, Bluetooth, in turn, was originally intended to replace cables between peripherals, using short range star connections that are not well suited for home automation due to its small coverage area, specially in non line of sight applications. The great strength of Wi-Fi and Bluetooth are their wide acceptance in the home automation market, since the great majority of the potential users of a home automation system already have several Bluetooth and Wi-Fi peripherals, so, Bluetooth or Wi-Fi products generally can be deployed in user's homes without the use of specialized network gateways, like in the case 802.15.4 products.

To address the low coverage disadvantage of its previous standard releases, Bluetooth Special Interest Group (SIG) published, in 2017, the Bluetooth Mesh Profile Specification [14]. This standard specifies a flooding mesh network over Bluetooth advertisement channels, this way, no modifications to the underlying Bluetooth Stack are necessary, which makes Bluetooth mesh profile independent of a certain version of Bluetooth Stack: any release higher than Bluetooth 4.0, that was the first release to support the Low Power Stack is compatible with the Mesh Profile. To allow backward compatibility and simplicity, the proposed standard relies in a routing 
TABLE I

COMPARISON BETWEEN IOT STANDARDS

\begin{tabular}{ccccc}
\hline Technology & Range & Topology & Power & Market \\
\hline Wi-Fi & Short & Star & High & Content Delivery \\
LoRaWAN & Long & Star & Low & Smart Cities \\
TSCH & Average & Mesh & Low & Industrial Applications \\
802.15.4 & Average & Mesh & Low & Home automation \\
Bluetooth & Average & Mesh & Low & Home automation \\
\hline
\end{tabular}

scheme that can suffer from broadcast storm problems [15], low scalability and low energy efficiency [16].

In Table I we summarize the characteristics of each mentioned protocol where we can see that the Bluetooth mesh profile have similar properties than the already established 802.15.4-2006 based protocols (Thread and Zigbee). This way, its is interesting to highlight the protocol advantages and address its weaknesses, aiding system designers to choose the most suited protocol for a certain application. In the next section, the efforts from industry and academia to evaluate and improve Bluetooth mesh networks will be presented.

\section{RELATED WORK}

The performance of standard Bluetooth Mesh was compared, using a real deployment with multi standard radios, with Thread and Zigbee in [16]. Results show that the Bluetooth SIG's proposed mesh protocol achieved lower Packet Throughput, and higher latency than 802.15 .4 based networks. The study also states that the performance degradation increases with the network size and is more pronounced when payloads greater than 8 bytes are used, which is the maximum Bluetooth Mesh payload without fragmentation. Other studies also assess Bluetooth mesh performance without comparing with a competing solution. In [17], Bluetooth mesh performance is evaluated using an experimental testbed, a statistical approach and a graph-based simulation model. It concludes that the technology have a lot of potential, but still has issues with energy efficiency because its focus is forming a backbone with energy unconstrained nodes. The studies motivates further research to improve the protocol performance. Some works suggests some improvements that do not break the compatibility with the current standard, like [18] and [19] which deeply studies reliability, delay, and scalability and them proposes only parameter changes that can improve the evaluated metrics. Another work [20] proposes the use of the extended advertising capabilities introduced with Bluetooth 5 to improve Bluetooth mesh performance.

The other research line that yields better performance Bluetooth mesh networks is proposing protocols that do not abide with the official standard released in 2017. Even prior that date, several works already explored the construction of Bluetooth Low Energy (BLE) Mesh Networks using some already established techniques employed in other types of wireless sensor networks. An exhaustive survey on the different approaches is available in [21] where several mesh proposals are discussed.

Some works try to bound the message repetition to improve energy efficiency and packet delivery ratio (PDR) in flooding routing, for example [22] proposed a mesh network based on the Gossip algorithm, where each node has a certain probability of retransmitting an overheard message, as opposed to simple flooding where the message is always retransmitted at least once. Using a similar message transmission bounding strategy the authors in [23] proposed a flooding routing Bluetooth Mesh network where the nodes first monitor the channel to check for message repetitions. A message is only retransmitted if it is not overheard for $c$ times during a variable time window, governed by the Trickle algorithm [24]. According to [23], this proposal increases the scalability and the packet delivery ratio when compared with conventional flooding.

Another open source Bluetooth Mesh protocol is FruityMesh [25], which is very different from the standard solution or the previous mentioned work in [23] since it is implemented as connection oriented mesh network forming a Bluetooth scatternet. Advertisement packets with cluster information are periodically broadcast and used by the nodes to find the most suitable cluster for network joining. The basic routing is by flooding each connection with received messages, on the other hand, this flooding does not interfere with packet delivery since connection oriented Bluetooth transmissions uses channel hopping time division multiplex.

As a motivation to check whether its better to deliver messages using advertisement channels or connection oriented connections, the authors from [26] compare the work from [23] with FruityMesh [25] using real hardware platforms. A 9 node network was tested with increasing packet generation rates. The study finds that network packet delivery ratio (PDR) decreases equally on both implementations. For efficiency metrics, delivery latency is significantly increased on Fruity Mesh, while stands unaffected on Trickle routing. On the other hand, Trickle routing requires three times more energy to generate the same number of packets, bearing the study inconclusive when indicating whether connection oriented Bluetooth possesses significant advantages over advertisement based networks.

Some other works propose Bluetooth Mesh Networks with routing algorithms, for instance [27] proposes a mesh network which uses the RPL protocol [28]. The proposed method was developed to work with the Android operating system and was evaluated using smartphones. For this reason, power consumption and latency are severely influenced by the chosen hardware and cannot be directly compared with other techniques. A similar approach was proposed in [29], but this time the implementation was verified using Raspberry Pi Boards and compared to 802.15.4 [13]. Results show that Bluetooth Mesh over RPL can outperform 802.15.4 in packet delivery ratio and duty cycle.

Another technique that can improve the performance of flooding networks is clustering. A clustered network is divided in several cells where one node assumes a special role of coordinating transmissions and forwarding aggregated data to the destination. This architecture can provide better energy efficiency and scalability by reducing the number of exchanged messages between sensors [30]. One of the first and most widely used clustering schemes is the Low Energy Adaptive 
Clustering Hierarchy (LEACH) [31]. The main objective of $\mathrm{LEACH}$ is to increase the network energy efficiency by randomly selecting an arbitrary proportion of network nodes to become these specialized nodes, denominated cluster heads (CHs) while the ordinary nodes associates to the closer $\mathrm{CH}$. This work proposes the application of LEACH technique in the standard Bluetooth mesh profile in order to increase its energy efficiency.

The contribution of this paper is twofold: first, we evaluate the energy efficiency and PDR of the Standard Bluetooth mesh protocol as a function of network size, traffic load and different network settings, concluding that flooding routing severely impacts both metrics due to excessive packet repetition and high idle listening times. Then, we select the most suited protocols to reduce the causes of the performance degradation. For reducing idle listening, the ContikiMAC protocol was applied [32] while the packet repetition reduction was achieved using a clustering scheme based on the LEACH [31] protocol. While the proposed techniques are not novel, to the best of our knowledge, it is the first time that they were applied in the Bluetooth Mesh network context. Their effectiveness were verified by computer simulations which show that, for dense networks, clustering can yield approximately $150 \%$ gain in PDR and energy efficiency, while radio duty cycling alone may yield up to $78 \%$ gain in energy efficiency for lightly loaded systems.

The remainder of this paper is organized as follows. Section III describes the Bluetooth Stack and the Official Bluetooth Mesh Profile. Section IV presents the techniques used to improve Bluetooth Mesh Performance. Section V describes the algorithms proposed in this manuscript. Section VI evaluates the effectiveness of the proposed algorithms using computer simulations. Finally, Section VII concludes the paper.

\section{Bluetooth LOW ENERGY}

The Bluetooth Protocol Stack [12] was first proposed in 1998 to replace wired connections between computers and devices. For this task it was first conceived as a piconet composed of a master and up to seven slaves. In its early years, the focus of the proposed protocol improvements was in range and transmission speed.

In 2010, the breakthrough 4.0 specification was released which introduced not only range/rate improvements to the current Bluetooth Standard, but a completely new Low Energy Stack (BLE Stack) that focuses in year long battery life using a single coin cell battery.

The BLE PHY layer divides the $2.4 \mathrm{GHz}$ Industrial Scientific and Medical (ISM) band in 40, $2 \mathrm{MHz}$ spaced channels. Gaussian frequency shift modulation is used to transmit with up to $2 \mathrm{Mbit} / \mathrm{s}$.

The Link Layer (LL) uses 37 of the defined channels for frequency hopping bidirectional communication between connected devices, while the remaining 3 channels are called advertisement channels used for device discovery, connection establishment and broadcast transmissions.

An unconnected BLE device uses advertisement packets to broadcast pairing information and presence for potential device users. Each transmission is performed in a successive manner in each advertisement channel, no synchronization is performed. Nearby scanning devices can use received advertisement information to start a connection with an advertiser device. Once connected, the advertiser joins the master/slave piconet that is coordinated by the scanning device.

The advertisement channels can also be used for sending data to an unpaired device. This feature is used as the base of the Bluetooth mesh protocol, that will be introduced in the next section.

\section{A. Bluetooth mesh}

The Bluetooth Mesh Profile [14] is a layered architecture released in 2017 to enable one-to-many multihop communication using the Bluetooth Low Energy Stack.

The first defined Mesh Layer (the Bearer layer) defines how messages are transported between nodes using an underlying BLE profile. Currently, two bearers are defined, the preferred transmission method is the Advertising Bearer which uses Bluetooth advertising and scanning facilities. There is also a connection oriented bearer (named GATT bearer) that is provided to enable backward compatibility with Bluetooth devices ta does not support the connectionless Bluetooth mesh profile, on the other hand, the main Bluetooth Mesh Transmission layer is the Advertisement Bearer and, for this reason, this work will focus on it.

A basic Bluetooth Mesh node implements two advertisement communication roles to form a flooding routing network. The first is the scanning role which continuously monitors the Bluetooth advertisement channels to intercept any mesh message sent by nearby nodes. Any received mesh message will be retransmitted in a successive manner after a random delay using the three advertisement channels. This way, the message can be received by other surrounding nodes which in turn can perform its own retransmission rounds. This process allows the message to propagate throughout the entire mesh network, eventually reaching its destination.

On top of the basic routing method, some logic must be added to avoid routing loops that can waste resources and congest the network with already transmitted packets [33]. First, every transmitted packet is stamped with a time to live (TTL) counter which is decremented for each retransmission. Only TTL $\geq 1$ messages will be retransmitted, avoiding infinite routing loops.

To optimize the initial TTL value for the message, each node periodically broadcasts a heartbeat message which contains the generator node address and the number of hops that the message experienced so far.

Upon reception of a heartbeat message, each node can update an internal table with the minimum hop count that is necessary to reach the heartbeat generator, and then rebroadcast the message with an updated hop count. The minimum hop count can be used to calculate the initial TTL of a message addressed to an specific node. If no minimal hop count data is available, a fixed value of up to 127 can be used for TTL.

Then the Bluetooth Mesh Profile defines four network features that may be implemented simultaneously, each of these functions will be detailed in the following paragraphs. 
The basic node which is always on and retransmits all the received messages is named Relay node. To further decrease the flooding congestion, each relay node maintains a broadcast message list. If a relay receives an already broadcast message, it is discarded without retransmission.

A relay node must scan the advertisement channels with a duty cycle as close to 100 percent as possible in order to avoid missing any incoming mesh messages, such behavior severely degrades its energy efficiency.

To avoid wasting energy resources, a Low Power Node (LPN) is designed to operate at significantly reduced duty cycles. It is allowed to turn off its receiving circuits and wake up periodically to scan for new messages.

Since it is highly likely that some messages addressed to a LPN will be transmitted when it is sleeping, a Friend Function is proposed. A node operating the Friend function is responsible for storing all incoming messages addressed to its associated LPNs. All LPN communication is routed through its associated Friend.

The low power nodes, in turn, issue a Poll Request message to its associated friend at the start of each active cycle. The answer should be a Poll Response packet containing the oldest stored message and the number of remaining stored messages addressed to the LPN.

The poll request/poll response process continues until no stored messages are available, at this point the LPN node returns to the sleep state, as illustrated in Fig. 1.

Since a LPN can only receive messages through the poll request/response mode, an unassociated LPN node will periodically broadcast a Friend Request message with TTL $=1$. The Friend nodes in range answer the Friend Request with a Poll Offer, which contains information like the Friend Available Memory, the size of the subscription list for the LPN and the number of Friend Offers that the Friend has sent. The low power node uses this information together with received signal strength to send a Friend Poll packet to the most suitable Friend which returns a Friend Update indicating that the Friendship was established.

The poll request generates some effects apart the obvious positive effects on battery life of the LPN nodes. There is the overhead caused by the extra Friendship establishment and the Poll Request/Response messages generated by nodes. On the other hand, all LPN/Friend messages are transmitted with $\mathrm{TTL}=1$ since both nodes must be in range to conform to the profile specification. In addition, a LPN node does not participate on the flooding routing process which can lower the network congestion but lower the packet delivery ratio for sparse networks as well. This non-trivial effect will be investigated in the Section VI.

Through this proposed scheme, the Bluetooth Special Interest Group (SIG) was able to propose a mesh network profile without changing the basic Bluetooth Low Energy Specification where addressed messages are only sent when devices are performing paired roles, on the other hand, since flooding routing relies on a somewhat uncontrolled repetition of messages, energy efficiency and congestion issues may arise.

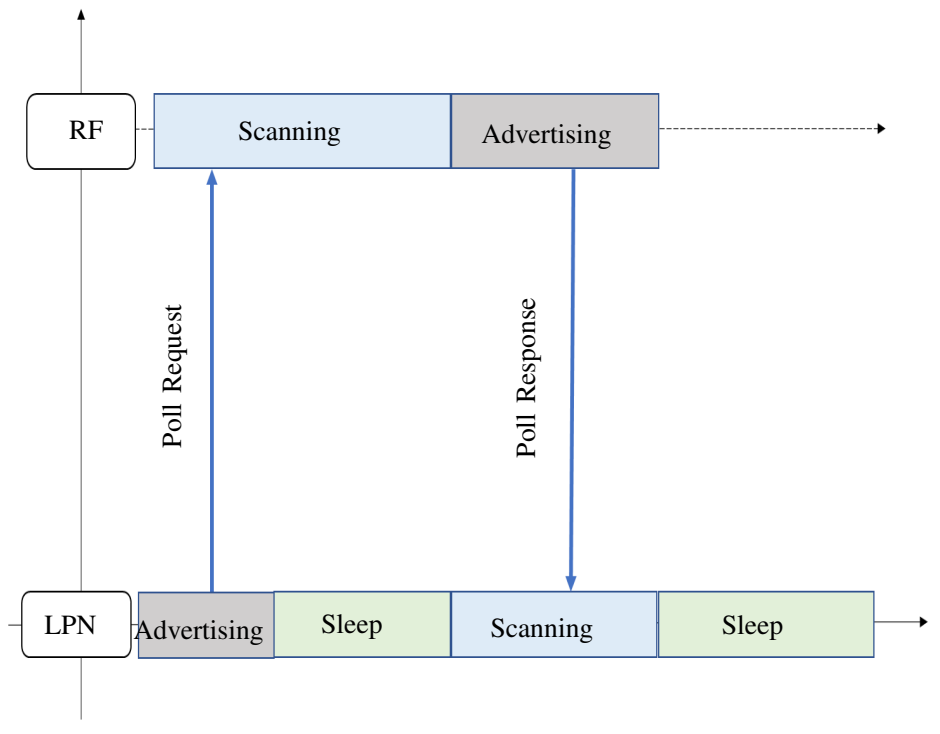

Fig. 1. The communication with poll request between Friend and LPN. A Low power node starts issuing a Poll request message right after entering active state. After a predetermined receive delay, the friend node sends a poll response, with the oldest data and the number of available messages for this LPN. Upon reception of the response, the Low power node may sleep or issue a new Poll Request if there are more available messages.

As an aggravating factor, the advertisement channels use a pure unsynchronized medium access. This fact degrades the packet delivery performance on dense deployments and requires that a basic mesh node must listen continuously when not transmitting, these factors severely decrease the battery life of real applications.

\section{IMPROVING FLOODING PERFORMANCE}

In this section, the recent community efforts to implement energy saving concepts that can be applied to Bluetooth mesh networks are reviewed and the methods that will be proposed in this work in order to improve the energy efficiency of standard Bluetooth mesh Networks are described.

\section{A. Clustering Algorithms}

A well-known approach to improve energy efficiency of flooding networks is using clustering schemes. In a clustered network, routing is performed in a hierarchical fashion where some nodes assume special roles (and are called Cluster Heads - CH). In contrast, flat routing schemes, like flooding routing used in Bluetooth Mesh, implements the same functions in each network node. Due to its architecture, hierarchical routing can provide better energy efficiency and scalability since it can reduce the number of exchanged packets between sensors. In addition, Bluetooth mesh nodes can already assume several roles which can be easily extended to support hierarchical routing.

One of the first and most widely used hierarchical routing scheme is called Low Energy adaptive clustering hierarchy (LEACH) [31]. Due its wide adoption and simple implementation, this technique will be used as a base of the 
proposed clustering scheme. The main objective of LEACH is to increase the network energy efficiency by randomly selecting an arbitrary proportion of network nodes to become cluster heads. The probability of a node becoming cluster head is specially developed to uniformly distribute energy consumption between nodes by periodically rotating cluster heads, since $\mathrm{CH}$ energy consumption is higher than ordinary nodes. Ordinary nodes will choose its cluster head based on received signal strength of messages originated by the $\mathrm{CHs}$. In the original $\mathrm{LEACH}$ [31], the $\mathrm{CHs}$ are also responsible in creating a Time Division Multiple Access (TDMA) schedule to coordinate the transmissions of the associated cluster nodes. This process can save a significant amount of energy since nodes that are not transmitting can stay in sleep state. Finally, it is CH's function to aggregate and transmit data received from their cluster members to the central node.

Several improvements were proposed for LEACH each of them focusing in different design goals. A detailed survey about LEACH modifications can be found in [30] and the variants that will be applied to the proposed algorithm will be briefly presented in the following paragraphs.

The authors in [34], [35] extend the LEACH protocol to allow multi hop communication between $\mathrm{CHs}$ and the central node and use slightly modified mathematical functions to better balance the $\mathrm{CH}$ distribution. Finally, in the cluster formation phase, each sensor node selects the $\mathrm{CH}$ that implies the lowest energy consumption while sending node data to the central node.

Another interesting proposal is E-LEACH [36] where the network operation starts as the original LEACH. As the network keeps operating, the residual energy of every sensor node is used as a parameter to choose the new CHs. Finally the C-LEACH (cell leach) [37] proposes a network partitioned into hexagonal cells which contain several nodes and a special cell head which is rotated periodically. Seven nearby cells form a cluster in the network, which contains one Cluster Head. A TDMA scheme manages data transmission between nodes and cell heads, and also between cell heads and cluster heads. According to the authors, this protocol has better network coverage, is highly scalable and energy efficient when compared to the original LEACH. On the other hand, this gain is accompanied of increased complexity and message overhead.

\section{B. Radio Duty Cycling Algorithms}

The simple flooding technique suffers from low packet delivery ratios and poor energy efficiency. The Bluetooth Mesh Profile Specification [14] states that a device should perform passive scanning with a duty cycle as close to 100 percent as possible in order to avoid missing incoming mesh messages. This activity is energy intensive, specially on cluster heads that must forward messages on behalf of all its cluster participants. To further decrease power consumption, the use of lower than $100 \%$ duty cycles may be helpful. In this regime, some messages may be lost since the nodes may be sleeping when source nodes are transmitting. To avoid this effect, a radio duty cycling algorithm must be used.
Radio duty cycling algorithms can be classified in asynchronous and synchronous. Examples of synchronous protocols are Bluetooth Low Energy operating in the MasterSlave mode and the IEEE Time Switching Channel Hopping [5]. These algorithms provide great robustness because they combine slotted contention free operation with channel hopping, at the cost of being more complex to operate due to synchronization efforts. Asynchronous algorithms, on the other hand, do not require a synchronization master and stringent timing requirements. This way they are easier to implement on less capable processors. One popular algorithm of this category is ContikiMAC [32] which defines in its operation a Channel Assessment Period $\left(t_{c}\right)$. Each receiver checks the channel for activity at each $t_{c}$ seconds, and each transmitter must repeat the message for at least $t_{c}$ seconds for broadcast messages or until acknowledgement (ACK) is received in the case of unicast transmissions. If a packet transmission is detected during a channel assessment, the receiver is kept on to be able to receive the packet. This timing guarantees that at least one transmission will be carried while the receivers are performing a channel assessment operation.

This duty cycling scheme can be effective in wireless sensor networks because several radios can save RX energy while only one transmitter must spend more TX energy [32], [38]. It is important to notice that on low power radios, TX power consumption is usually similar to RX power consumption. On the other hand, this process can increase congestion in flooding networks since the number of transmitted messages increases with the decreasing duty cycle. This effect will be investigated in Section VI.

In the next section, we propose some adaptation to Bluetooth Mesh protocol to include radio duty cycling and clustering techniques presented in Section IV.

\section{Proposed Algorithm}

Since flooding networks suffer from low packet delivery ratios and low energy efficiency, an extension of BLE Mesh operation is proposed. Then, in the next sections, the performance of the extensions is numerically evaluated through computer simulations.

The first proposed measure to increase the energy efficiency of Bluetooth Mesh is Radio Duty Cycling. In this work, we use the simplified ContikiMAC [32] mechanism, since, as explained in Section IV-B, channel assessment is not always available in standard Bluetooth commands. In this case, the receiving duty cycle must be lowered to an arbitrary value and a number of spaced repetitions must be made to guarantee that at least one message is sent while the receiving radios are listening. As an example, for a $50 \%$ duty cycle, two repetitions are carried within the one cycling period, this way at least one message will be transmitted when the receiver is active. If the listening duty cycle is lowered to $25 \%$, then 4 transmissions within the duty cycle must be performed. This process is illustrated in Fig 2 where the TX and RX windows of two radios operating at different duty cycles is shown. It is worthy to note that the interval between two consecutive transmissions is smaller than the RX window size, so it is impossible to fit 


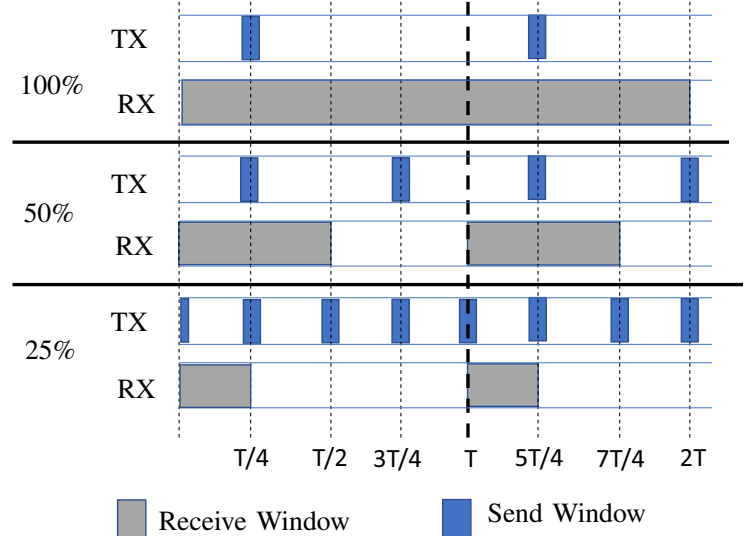

Fig. 2. Proposed duty-cycling scheme.

a RX window in a region without at least one TX event. This guarantees connectivity for radios with lower than $100 \%$ duty cycle listening windows without requiring synchronization.

The second proposed measure to improve energy efficiency and packet delivery ratio is limiting the number of forwarded packets [24]. This can be specially beneficial in highly congested networks where simple flooding produces excessive packet redundancy, which increases collisions. Since low power nodes do not forward mesh packets, we introduce a geographical C-LEACH [37] variant where the cluster head is configured as a Relay-Friend node where all other cluster members are configured as low power nodes.

The Relay Friend Cluster Head is rotated whenever the current head reaches a configurable energy consumption. The node with higher amount of residual energy among cluster LPNs becomes the new cluster head. The Poll Request/Response protocol is extended to include cluster management fields. The residual energy of each LPN is sent on poll request messages, while the Poll Response message now carries a field indicating whether the node must become the new cluster head. Since these messages must already be exchanged between LPNs and friends for friendship operation, cluster management overhead is very low when compared to unclustered Bluetooth Mesh networks.

In this preliminary study, each node have prior information about its geographical location, which is used to initially form the clusters. The first cluster head is chosen randomly.

\section{Numerical RESUlts}

In this section we present some numerical results obtained from a Bluetooth network simulator programmed using the SimPy discrete-event simulation framework [39]. Propagation model was based on Cooja Unit Disk Graph Radio Medium [40] where only large scale path loss is considered for decoding and interference. The considered path loss coefficient is $\alpha=2.08$, and radio parameters are based on Nordic Semiconductor nRF51822 [41] Bluetooth System on a Chip (SoC), which are reproduced on Table II. Radio reception is considered successful when the received power is above
nRF51822 sensitivity and no other transmission above the sensitivity range were received at current channel during the whole duration of packet transmission.

TABLE II

SYSTEM PARAMETERS

\begin{tabular}{ccc}
\hline Parameter & Value & Unit \\
\hline Path Loss Exponent & 2.08 & - \\
Radio Sensitivity & -93.0 & $\mathrm{dBm}$ \\
Transmission Power & 4.0 & $\mathrm{dBm}$ \\
SoC Voltage & 3.0 & $\mathrm{~V}$ \\
SoC TX Current & 10.5 & $\mathrm{~mA}$ \\
SoC RX Current & 13.0 & $\mathrm{~mA}$ \\
SoC Processor Current & 4.8 & $\mathrm{~mA}$ \\
SoC Sleep Current & 2.8 & $\mu \mathrm{A}$ \\
Transmission Frequency & 2.4 & $\mathrm{GHz}$ \\
\hline
\end{tabular}

Each packet was transmitted sequentially using the advertisement bearer through Bluetooth channels 37 to 39. All routing rules from Bluetooth Mesh Profile [14] were implemented, according to the node's configured network features. Unless otherwise specified, time to live values for each message were optimized using prior received heartbeat messages, which are periodically broadcast by each node.

Fig. 3 shows Packet Delivery ratio as a function of Network Size for different TTL values and with $95 \%$ confidence intervals. In this network $25 \%$ of the network components are configured as Relay-Friend Nodes, $75 \%$ are Low Power Nodes. Packet generation of each node follows a Poisson distribution with 10 second average. The nodes are stationary and randomly distributed in a rectangular area with $(22 \times i)$ square meters, where $i$ is the total number of nodes for the simulation. Each simulation is split in four rounds with different random positioning. The simulation runs until each node sends at least 70 packets. From Fig. 3 it can be seen that the packet delivery ratio decreases with the network size. This phenomena is caused by two combined effects: first the average hop count between source and destination grows with network size, this way, when the nodes are configured with a too low initial TTL values, more messages are dropped in route due to insufficient TTL; second if a higher than necessary TTL is used, excessive flooding is generated, which increases collision events. It can also be observed that increasing TTL, even using information of the minimum hop count between source and destination generally has little positive effect on packet delivery ratio since collision outages increases with TTL in a rate that nullifies the effect on low TTL outages. As a partial conclusion, since TTL optimization minimum is not sufficient to control Bluetooth mesh flooding, which may improve network scalability, other methods must be investigated.

To complement the results from the previous experiment, Fig. 4 shows the packet delivery ratio (\%) as a function of the per node average time between packet generation for a 16 nodes network with TTL $=20$. It can be observed that packet delivery ratio increases with the average packet generation period, exceeding $99 \%$ when this period is greater than $10 \mathrm{~s}$. On the other hand, when the packet generation rate 


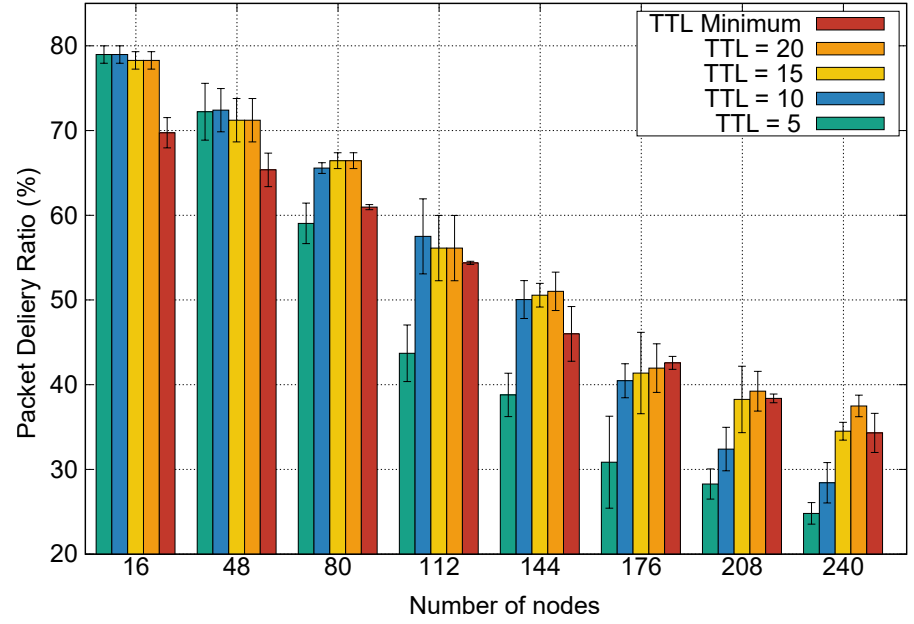

Fig. 3. PDR (\%) vs Network Size for different TTL values. Average per node packet generation ratio: $10 \mathrm{~s}$.

is increased, flooding routing rapidly saturates the transmission channels which decrease the packet delivery ratio due to collision events. In addition, the packet replication needed for flooding routing raises concerns about energy efficiency. For this reason, Fig. 5 shows the energy efficiency as a function of the number of nodes. It can be observed that the energy efficiency is strongly correlated with Packet Delivery Ratio (Fig. 3). One may argue that energy efficiency must decrease when the number of nodes or the initial TTL of the network increases since extra message replication is generated. This behavior is not observed because total energy consumption of a Bluetooth mesh node is dominated by idle listening time which is much bigger than transmission time as observed at Fig. 6 . This behavior motivates the use of idle listening reduction techniques like the ones used in this work: radio duty cycling and clustering. The effectiveness of the proposed solutions will be investigated in the following tests.

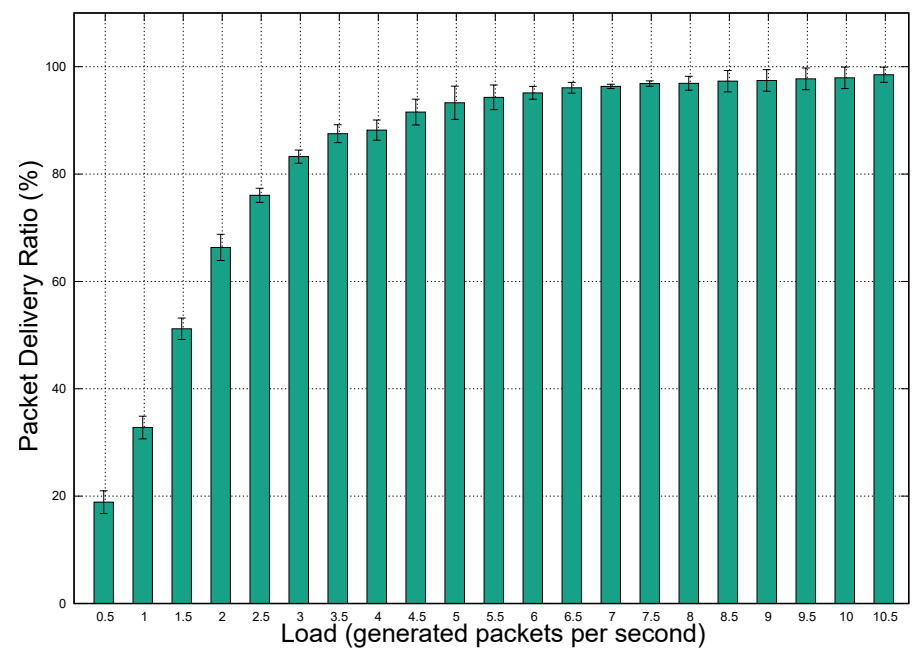

Fig. 4. PDR(\%) vs average time (in seconds) between per node packet generation. Network size is $16, \mathrm{TTL}=20$.

Fig. 7 shows packet delivery for different duty cycles as

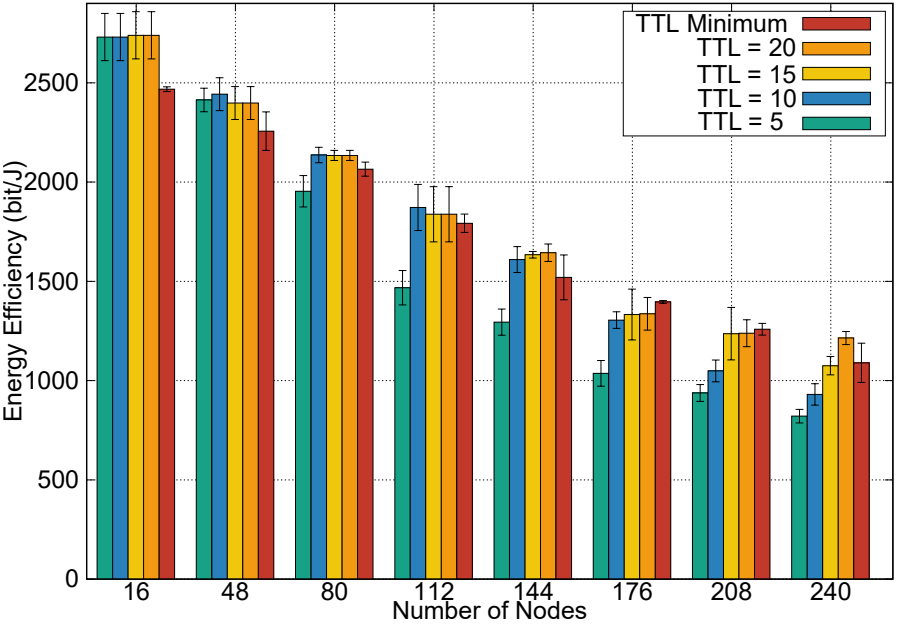

Fig. 5. Energy efficiency of Bluetooth Mesh Network as a function of network size. Average per node packet generation interval is $10 \mathrm{~s}$.

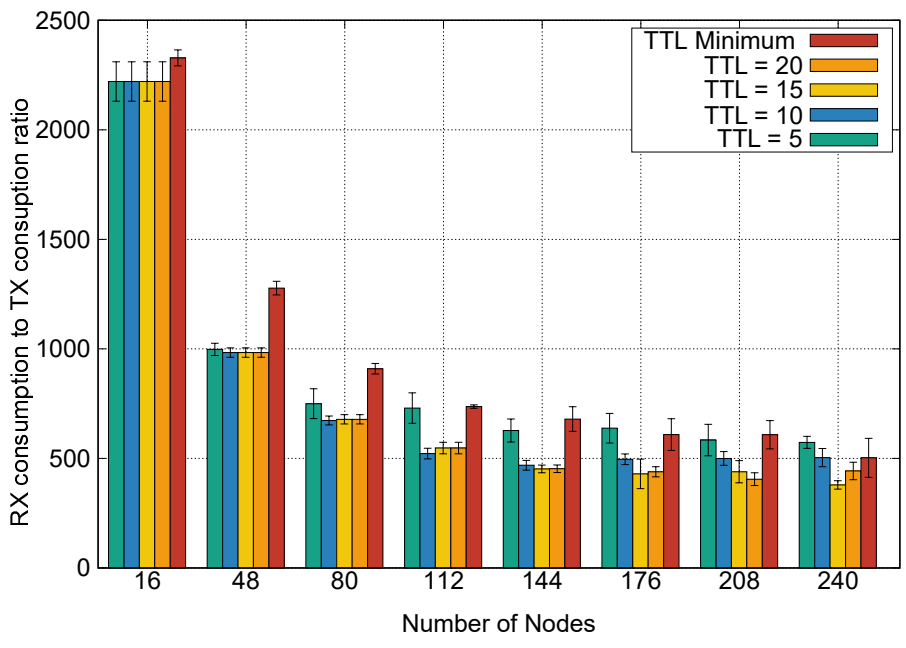

Fig. 6. Energy efficiency of Bluetooth Mesh Network as a function of network size. Average per node packet generation interval is $10 \mathrm{~s}$.

a function of network size for a network with $75 \%$ of LPN nodes and $25 \%$ of Relay-friend nodes with TTL $=20$ and $10 \mathrm{~s}$ average packet generation interval. It also can be observed that the PDR is more negatively affected in networks with lower duty cycles because they are more susceptible to collision outages when the network size increases. This effect is observed because the chosen duty cycling technique transmits a rapid sequence of packet copies, which can increment the network traffic, which in turn results in higher collision rates. On the other hand, Fig. 8 show the energy efficiency of the same network operating in duty cycled regime. It can be observed that duty cycling brings up to $78 \%$ of energy efficiency improvement for duty cycle of $25 \%$ compared with $100 \%$. In this case, the simulation brings for duty cycle $=25 \%$ $4875 \mathrm{bit} / \mathrm{J}$ for 16 nodes while at the pure Bluetooth is $2730 \mathrm{bit} / \mathrm{J}$ for 16 nodes. For duty cycle of $50 \%$, the best results is for 48 nodes. In this case, for $50 \%$ of duty cycle there is $2998 \mathrm{bit} / \mathrm{J}$ and for $100 \%$ is 2442 and this results in $22 \%$ of energy efficiency improvement. For small networks, 
the best strategy is choosing lower duty-cycles but when the network size increases, PDR degradation for low duty cycle networks impacts energy efficiency, making high duty cycle networks more energy efficient.

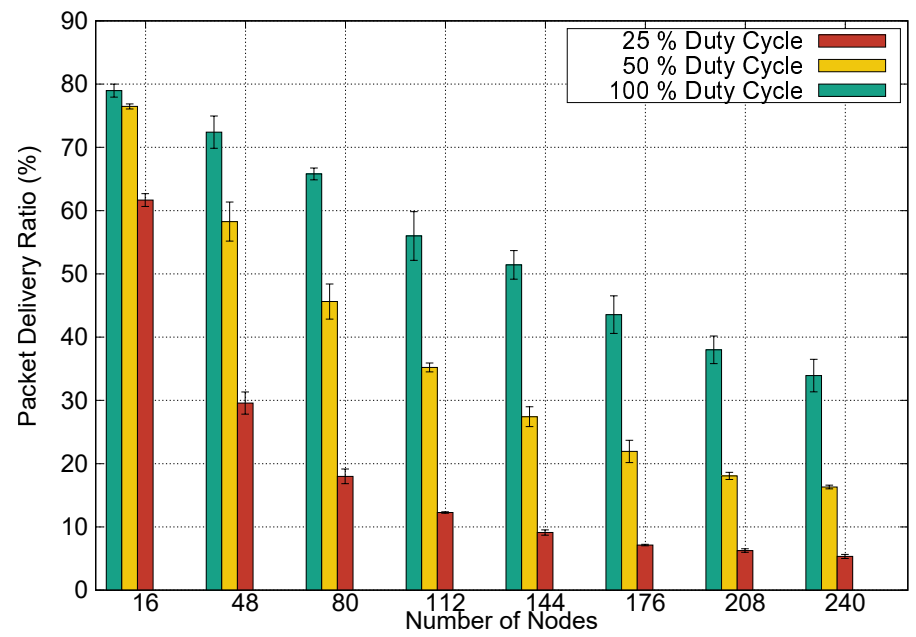

Fig. 7. Packet delivery ratio for different radio duty cycles as a function of network size. TTL $=20$, average packet generation rate $=10 \mathrm{~s}$. Network composed from $75 \%$ of LPN's and $25 \%$ of Relay-Friend

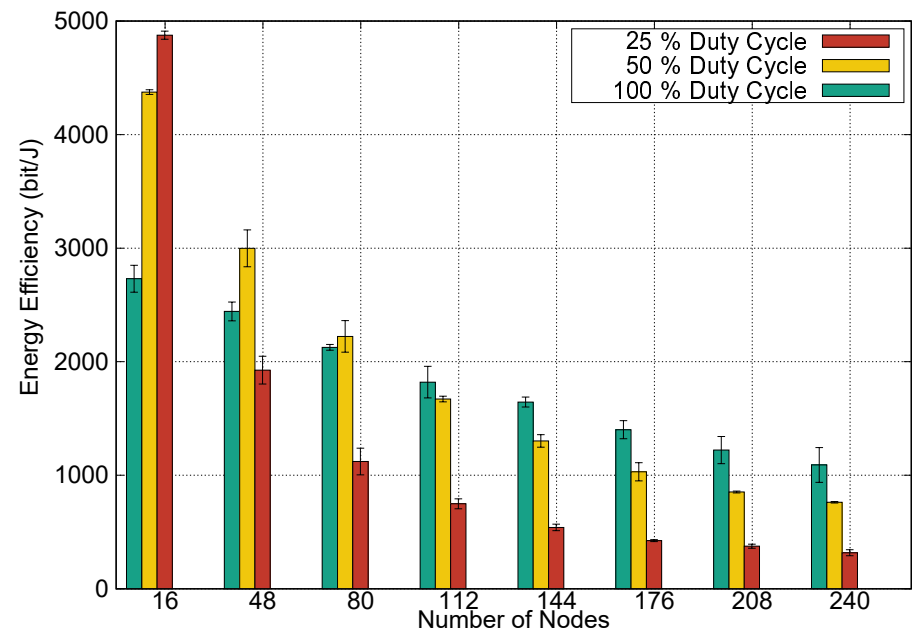

Fig. 8. Energy efficiency for different duty cycles as a function of network size. Average Packet generation rate is $10 \mathrm{~s}$.

On the other hand, with networks with up to 48 nodes and lower duty cycles, we have more energy efficiency gains than packet delivery ratio degradation, this way the use of radio duty cycling may be justified. Even with an increased number of packet transmission retries an improvement in energy efficiency can be attained. Another advantage of radio duty cycling technique is that no modification in the current Bluetooth Mesh Profile is needed, only a simple modification on the packet transmission logic.

If protocol modifications are allowed, the clustering protocol proposed in Section $\mathrm{V}$ can be used to limit the packet replication that degrades the lower duty cycles networks. These results are presented in the next experiments where a cluster head relay friend node (CHRF) was added for each
3 low power nodes of the network. For a fair comparison, the unclustered tests are performed with a network with the same proportion of relay friends and low power nodes. Packet generation rate is set to $10 \mathrm{~s}$ and the simulation stops when the first node depletes its battery.

Fig. 9 shows the packet delivery ratio and Fig. 10 shows the energy efficiency of clustered networks as a function of network size. It can be seen from both figures that the choice of the most suited technique depends on the network size and whether the deployment priority is energy efficiency or packet delivery ratio. When compared to conventional $100 \%$ duty cycle networks, the proposed clustering and duty cycle reducing techniques have a negative impact on packet delivery ratio for networks with less than 288 nodes.

On the other hand, in the same configuration, energy efficiency gains may be obtained. For small sized networks with up to 16 nodes, the best strategy is using the unclustered network with $25 \%$ duty cycle, which can attain $78 \%$ improvement when compared to the Baseline profile.

For big sized networks ranging from 288 to 720 nodes, the most energy efficient strategy is the unclustered $50 \%$ duty cycle networks which can provide up to $130 \%$ for 720 nodes. Clustered networks are suited for larger deployments.

For networks with more than 288 nodes, the clustered strategy presents the best energy efficiency, and for networks with more than 288 nodes, clustered networks also presents the best packet delivery ratio, which indicates that the technique is effective in preventing excessive packet replication which increases the network scalability and the energy efficiency. For less dense the proposed radio duty cycling reducing technique alone is enough to attain energy efficiency.

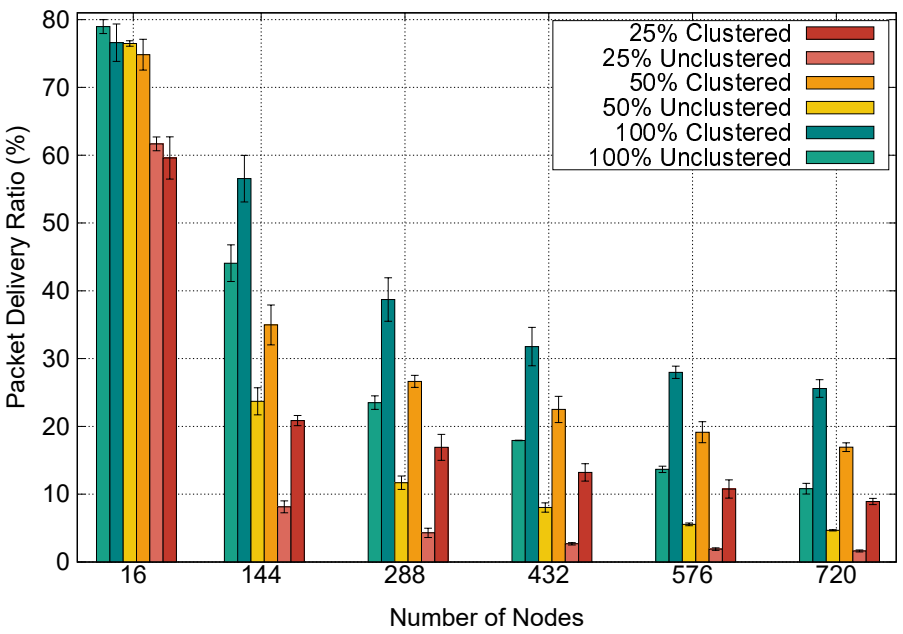

Fig. 9. Packet delivery ratio as a function of network density for Clustered Bluetooth Mesh and different radio duty cycles. TTL $=20$. Average packet generation rate is $10 \mathrm{~s}$.

\section{CONCLUSION}

This work proposed a Clustering Algorithm based on LEACH to improve the energy efficiency of Bluetooth Mesh networks. To further reduce the burden over the Cluster Heads, an Radio Duty Cycling algorithm inspired on ContikiMAC 


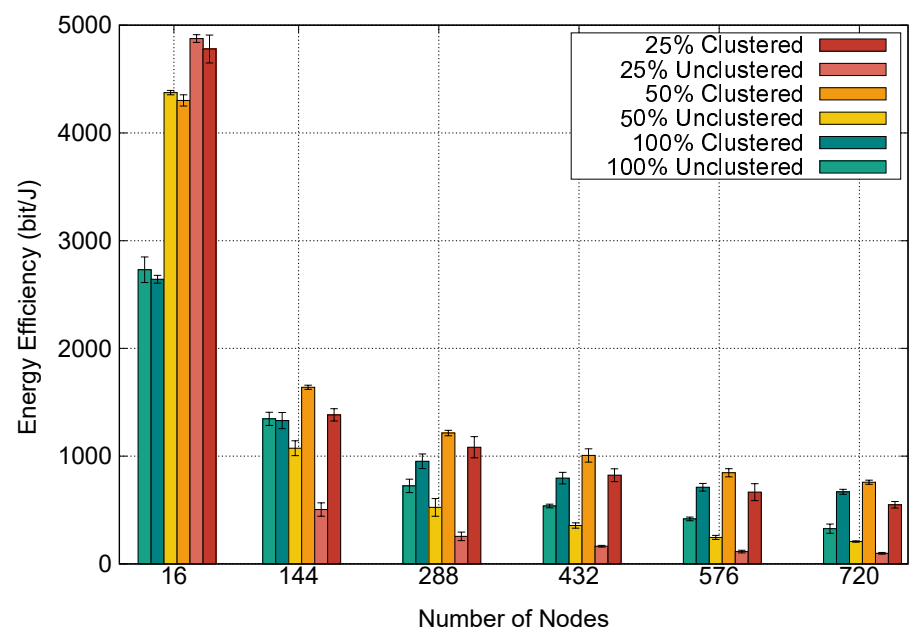

Fig. 10. Energy efficiency of Clustered Bluetooth Mesh as a function of network density for different duty cycles. TTL $=20$. Average packet generation rate $=10 \mathrm{~s}$.

as proposed. The effectiveness of the proposed solution was evaluated using computer simulations.

The paper shows that the energy efficiency of Bluetooth mesh networks is dominated by idle listening time of relay nodes which is much bigger than transmission times. This way radio duty cycling and clustering methods for reducing idle listening are very effective in improving energy efficiency.

It was observed that duty cycling brings up to $78 \%$ of energy efficiency improvement for a 16 node network operating with $25 \%$ duty-cycle and $60 \%$ for a $50 \%$ duty-cycle. Another advantage of radio duty cycle technique is that no modification in the current Bluetooth Mesh Profile is needed, only a simple modification on the packet transmission logic.

In addition, the proposed clustering technique has a slightly negative impact on packet delivery ratio of Bluetooth mesh networks for small networks while bringing positive impacts on energy efficiency and network lifetime. Moreover, clustered networks are less affected by collision outages, so that a steady packet delivery ratio for all network densities is sustained while the unclustered networks suffer from rapid degradation in packet delivery ratio when the network size increases.

As conclusion, it can be observed that the proposed clustering algorithm, when applied with the proposed radio duty cycling algorithm can provide higher efficiency energy when compared to the unmodified Bluetooth Mesh and the dutycycle technique.

Furthermore the study indicates that the results can be expanded for even bigger Bluetooth Mesh Networks, since clustering is effective in controlling the excessive message replication that is inherent in flooding operation.

\section{REFERENCES}

[1] L. Columbus, "2018 roundup of internet of things forecasts and market estimates," Dec 2018. [Online]. Available: https://www.forbes.com/sites/louiscolumbus/2018/12/13/ 2018-roundup-of-internet-of-things-forecasts-and-market-estimates/ \#1568d7327d83
[2] "IDC forecasts worldwide technology spending on the Internet of Things to reach $\$ 1.2$ trillion in 2022." [Online]. Available: https://www.idc.com/getdoc.jsp?containerId=prUS43994118

[3] "Internet of Things forecast - Ericsson mobility report," Jun 2019. [Online]. Available: https://www.ericsson.com/en/mobility-report/ internet-of-things-forecast

[4] J. A. Stankovic, "Research directions for the internet of things," IEEE IoT J., vol. 1, no. 1, pp. 3-9, Feb 2014. doi: 10.1109/JIOT.2014.2312291

[5] "IEEE Standard for Local and metropolitan area networks-Part 15.4: Low-Rate Wireless Personal Area Networks (LR-WPANs) Amendment 1: MAC sublayer," IEEE Std 802.15.4e-2012 (Amendment to IEEE Std 802.15.4-2011), pp. 1-225, April 2012. doi: 10.1109/IEEESTD.2012.6185525

[6] X. Vilajosana, Q. Wang, F. Chraim, T. Watteyne, T. Chang, and K. S. J. Pister, "A realistic energy consumption model for TSCH networks," IEEE Sens. J., vol. 14, no. 2, pp. 482-489, Feb 2014. doi: 10.1109/JSEN.2013.2285411

[7] N. Sornin and A. Yegin, "Lorawan ${ }^{\circledR}$ specification v1.1: Lora alliance ${ }^{\mathrm{TM}}$," Oct 2017. [Online]. Available: https://lora-alliance.org/ resource-hub/lorawanr-specification-v11

[8] "SigFox - the global communications service provider for the internet of things (IoT)." [Online]. Available: https://www.sigfox.com/en

[9] "Thread Group," https://www.threadgroup.org/, [Online; accessed 13October-2019].

[10] “ZigBee specifications "ZigBee Document 053474r15"," 2007, [Online; accessed 27-July-2019].

[11] "IEEE Standard for Information technology-Telecommunications and information exchange between systems - Local and metropolitan area networks-Specific requirements - part 11: Wireless LAN Medium Access Control (MAC) and Physical Layer (PHY) Specifications Amendment 2: Sub $1 \mathrm{GHz}$ License Exempt Operation," IEEE Std 802.11ah-2016 (Amendment to IEEE Std 802.11-2016, as amended by IEEE Std 802.11ai-2016), pp. 1-594, May 2017. doi: 10.1109/IEEESTD.2017.7920364

[12] "Bluetooth core specification version 5.3," https://www.bluetooth.com/ specifications/specs/core-specification/, [Online; accessed 04-august2021].

[13] "IEEE standard for information technology- local and metropolitan area networks- specific requirements- part 15.4: Wireless medium access control (MAC) and physical layer (PHY) specifications for low rate wireless personal area networks (WPANs)," IEEE Std 802.15.4-2006 (Revision of IEEE Std 802.15.4-2003), pp. 1-320, Sep. 2006. doi: 10.1109/IEEESTD.2006.232110

[14] "Mesh profile 1.0.1," https://www.bluetooth.com/specifications/specs/ mesh-profile-1-0-1/, [Online; accessed 04-august-2021].

[15] Hao Zhang and Zhong-Ping Jiang, "Performance analysis of broadcasting schemes in mobile ad hoc networks," IEEE Commun. Lett., vol. 8, no. 12, pp. 718-720, Dec 2004. doi: 10.1109/LCOMM.2004.837658

[16] AN1142: Mesh Network Performance Comparison, Silicon Laboratories, inc., 1 2018, rev. 0.2. [Online]. Available: https://www.silabs.com/documents/public/application-notes/ an1142-mesh-network-performance-comparison.pdf

[17] M. Baert, J. Rossey, A. Shahid, and J. Hoebeke, "The bluetooth mesh standard: An overview and experimental evaluation," Sensors, vol. 18, no. 8, 2018. doi: 10.3390/s18082409. [Online]. Available: https://www.mdpi.com/1424-8220/18/8/2409

[18] R. Rondón, A. Mahmood, S. Grimaldi, and M. Gidlund, "Understanding the performance of bluetooth mesh: Reliability, delay, and scalability analysis," IEEE Internet of Things Journal, vol. 7, no. 3, pp. 20892101, 2020. doi: 10.1109/JIOT.2019.2960248

[19] A. Hernández-Solana, D. Pérez-Díaz-De-Cerio, M. García-Lozano, A. V. Bardají, and J.-L. Valenzuela, "Bluetooth mesh analysis, issues, and challenges," IEEE Access, vol. 8, pp. 53784-53800, 2020. doi: 10.1109/ACCESS.2020.2980795

[20] D. Pérez-Díaz-De-Cerio, A. Hernández-Solana, M. García-Lozano, A. V. Bardají, and J.-L. Valenzuela, "Speeding up bluetooth mesh," IEEE Access, vol. 9, pp. 93267-93 284, 2021. doi: 10.1109/ACCESS.2021.3093102

[21] S. M. Darroudi and C. Gomez, "Bluetooth low energy mesh networks: A survey," in Sensors, 2017.

[22] P. Kyasanur, R. R. Choudhury, and I. Gupta, "Smart gossip: An adaptive gossip-based broadcasting service for sensor networks," in 2006 IEEE International Conference on Mobile Ad Hoc and Sensor Systems, Oct 2006. doi: 10.1109/MOBHOC.2006.278671. ISSN 2155-6806 pp. 91100 . 
[23] A. Gogic, A. Mujcic, S. Ibric Hodzic, and N. Suljanovic, "Performance analysis of bluetooth low energy mesh routing algorithm in case of disaster prediction," World Academy of Science, Engineering and Technology, International Journal of Computer, Electrical, Automation, Control and Information Engineering, vol. 10, pp. 929-935, 012016.

[24] P. Levis, N. Patel, D. Culler, and S. Shenker, "Trickle: A self-regulating algorithm for code propagation and maintenance in wireless sensor networks," in Proceedings of the 1st Conference on Symposium on Networked Systems Design and Implementation - Volume 1, ser NSDI'04. Berkeley, CA, USA: USENIX Association, 2004, pp. 2-2. [Online]. Available: http://dl.acm.org/citation.cfm?id=1251175.1251177

[25] R. Dvinge, A. Stalmach, and L. Nalpantidis, "Connection-based bluetooth mesh network as a low energy solution for off-grid data networks," 05 2019. doi: 10.1109/MOCAST.2019.8741589 pp. 1-6.

[26] Y. Murillo, B. Reynders, A. Chiumento, S. Malik, P. Crombez, and S. Pollin, "Bluetooth now or low energy: Should BLE mesh become a flooding or connection oriented network?" in 2017 IEEE 28th Annual International Symposium on Personal, Indoor, and Mobile Radio Communications (PIMRC), Oct 2017. doi: 10.1109/PIMRC.2017.8292705. ISSN $2166-9589$ pp. 1-6.

[27] S. Sirur, P. Juturu, H. P. Gupta, P. R. Serikar, Y. K. Reddy, S. Barak, and B. Kim, "A mesh network for mobile devices using bluetooth low energy," in 2015 IEEE SENSORS, Nov 2015. doi: 10.1109/ICSENS.2015.7370451 pp. 1-4.

[28] R. Alexander, A. Brandt, J. Vasseur, J. Hui, K. Pister, P. Thubert, P. Levis, R. Struik, R. Kelsey, and T. Winter, "RPL: IPv6 Routing Protocol for Low-Power and Lossy Networks," RFC 6550, Mar. 2012. [Online]. Available: https://rfc-editor.org/rfc/rfc6550.txt

[29] T. Lee, M. Lee, H. Kim, and S. Bahk, "A synergistic architecture for rpl over ble," in 2016 13th Annual IEEE International Conference on Sensing, Communication, and Networking (SECON), June 2016. doi: 10.1109/SAHCN.2016.7732968 pp. 1-9.

[30] S. K. Singh, P. Kumar, and J. P. Singh, "A survey on successors of leach protocol," IEEE Access, vol. 5, pp. 4298-4328, 2017. doi: 10.1109/ACCESS.2017.2666082

[31] W. R. Heinzelman, A. Chandrakasan, and H. Balakrishnan, "Energyefficient communication protocol for wireless microsensor networks," in Proceedings of the 33rd Annual Hawaii International Conference on System Sciences, Jan 2000. doi: 10.1109/HICSS.2000.926982 pp. 10 pp. vol.2-.

[32] A. Dunkels and adam@sics.se, "The contikimac radio duty cycling protocol," Tech. Rep. T2011:13, 2011.

[33] L. Tian, "A multi-flooding data transmission mechanism for lowduty-cycle wireless sensor network," in The 26th Chinese Control and Decision Conference (2014 CCDC), May 2014. doi: 10.1109/CCDC.2014.6852529. ISSN 1948-9439 pp. 2181-2184.

[34] A. Depedri, A. Zanella, and R. Verdone, "An energy efficient protocol for wireless sensor networks," 032003.

[35] C. Buratti, A. Giorgetti, and R. Verdone, "Cross-layer design of an energy-efficient cluster formation algorithm with carrier-sensing multiple access for wireless sensor networks," EURASIP Journal on Wireless Communications and Networking, vol. 2005, 01 2005. doi: 10.1155/WCN.2005.672

[36] M. Bani Yassein, A. Al-zou'bi, Y. Khamayseh, and W. Mardini, "Improvement on leach protocol of wireless sensor network (vleach)," JDCTA, vol. 3, pp. 132-136, 01 2009. doi: 10.1109/SENSORCOMM.2007.4394931

[37] A. Yektaparast, F. Nabavi, and A. Sarmast, "An improvement on leach protocol (cell-leach)," in 2012 14th International Conference on Advanced Communication Technology (ICACT), Feb 2012. ISSN 17389445 pp. 992-996.

[38] Y. Sun, O. Gurewitz, S. Du, L. Tang, and D. B. Johnson, "Adb: An efficient multihop broadcast protocol based on asynchronous duty-cycling in wireless sensor networks," 01 2009. doi: 10.1145/1644038.1644044 pp. 43-56.

[39] "Simpy," https://simpy.readthedocs.io/en/latest/, 2018, [Online; accessed 06-April-2019].

[40] F. Osterlind, A. Dunkels, J. Eriksson, N. Finne, and T. Voigt, "Crosslevel sensor network simulation with cooja," in Proceedings. 2006 31st IEEE Conference on Local Computer Networks, 2006. doi: 10.1109/LCN.2006.322172 pp. 641-648.

[41] "nRF51822," https://www.nordicsemi.com/Products/ Low-power-short-range-wireless/nRF51822, [Online; accessed 13October-2019].

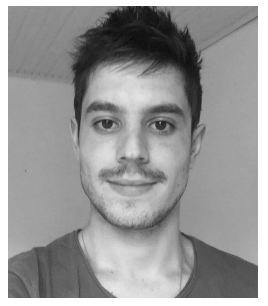

Joelton Deonei Gotz was born in Erechim, Brazil, in 1992. He obtained the B.S. degree in control and automation Engineering from the Federal University of Technology - Paraná(UTFPR), Brazil, in 2017. He received the M.Sc. in Electrical Engineering from the Federal University of Technology Paraná(UTFPR), Brazil, in 2020. Since August 2020, Joelton Deonei Gotz is currently a Ph.D student at UTFPR in the area of mechanical engineering. His research interests include Internet of Things (IoT), Machine Learning, product development.

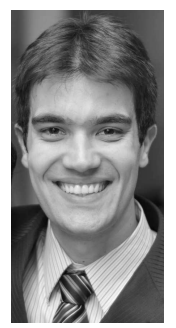

Ohara Kerusauskas Rayel (S'13-M'17) was born in Curitiba-PR, Brazil, in 1984. He received the B.Sc., M.Sc. and D.Sc. degrees in electrical engineering from the Federal University of TechnologyParaná (UTFPR), Curitiba, Brazil, in 2006, 2013 and 2015 respectively. He is currently an Assistant Professor at the same University. His research interests include cooperative communications, MIMO, energy efficiency and internet of things protocols.

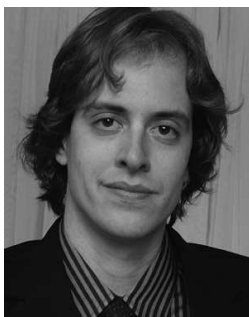

Guilherme Luiz Moritz was born in Gaspar, Brazil, in 1984. He received the B.Sc. and the D.Sc. degrees in electrical engineering from the Federal University of Technology-Paraná (UTFPR), Curitiba, Brazil, in 2012 and 2015, respectively, where he is currently an Assistant Professor of embedded systems, control systems, and wireless sensor networks. His research interests are in the area of wireless communications, cooperative communications, and the Internet of Things. 